\title{
Spin-glass freezing in Kondo-lattice compounds
}

\author{
Alba Theumann \\ Instituto de Física - UFRGS, Av. Bento Gonçalves 9500, 91501-970 Porto Alegre, RS, Brazil \\ B. Coqblin \\ Laboratoire de Physique des Solides, Université Paris - Sud, 91405 Orsay Cedex, France
}

S. G. Magalhães and A. A. Schmidt

Departamento de Matemática, Universidade Federal de Santa Maria, 97119-900 Santa Maria, RS, Brazil

(Received 25 May 2000; published 4 January 2001)

\begin{abstract}
A theory is presented that describes a spin-glass phase at finite temperatures in Kondo-lattice systems with an additional Ruderman-Kittel-Kasuya-Yosida interaction represented by long range, random couplings among localized spins as in the Sherrington-Kirkpatrick (SK) spin-glass model. The problem is studied within the functional integral formalism where the spin operators are represented by bilinear combinations of fermionic (anticommuting) Grassmann variables. The Kondo and spin-glass transitions are both described with the mean-field-like static ansatz that reproduces good results in the two well-known limits. At high temperatures and low values of the Kondo coupling there is a paramagnetic (disordered) phase with vanishing Kondo and spin-glass order parameters. By lowering the temperature, a second order transition line is found at $T_{\mathrm{SG}}$ to a spin-glass phase. For larger values of the Kondo coupling there is a second order transition line at roughly $T_{k}$ to a Kondo ordered state. For $T<T_{\mathrm{SG}}$ the transition between the Kondo and spin-glass phases becomes first order.
\end{abstract}

DOI: 10.1103/PhysRevB.63.054409

PACS number(s): 75.10.Nr, 05.50.+q, 64.60.Cn

\section{INTRODUCTION}

The antiferromagnetic $\mathrm{s}-\mathrm{f}$ exchange coupling of conduction electrons to localized spins in heavy fermion rare-earth systems is responsible for two competing effects: the screening of the localized moments due to the Kondo effect and the Ruderman-Kittel-Kasuya-Yosida (RKKY) interaction among magnetic impurities which may induce a long-range magnetic (antiferromagnetic or ferromagnetic) ordering or eventually a spin-glass magnetic ordering. The Doniach diagram $^{1}$ gives a good description of this competition: the Neel temperature $T_{N}$ is firstly increasing with increasing the absolute value of the exchange interaction constant $J_{k}$, then it is passing through a maximum and finally it tends to zero at the "quantum critical point"' (QCP). Such a decrease of $T_{N}$ down to the QCP has been observed in many cerium compounds, such as $\mathrm{CeAl}_{2}, \mathrm{CeAg}$, or $\mathrm{CeRh}_{2} \mathrm{Si}_{2}$, under pressure. Above the QCP, there exists a very strong heavy fermion character, but several possible behaviors, i.e., the classical Fermi liquid one with eventually a reduced Kondo temperature 2,3 or different non-Fermi-liquid (NFL) ones, have been observed in cerium or ytterbium compounds. ${ }^{4,5}$

But, in the case of disordered cerium alloys, the disorder can yield a spin-glass (SG) phase in addition to the NFL behavior at low temperatures around the QCP. The magnetic phase diagram of $\mathrm{CeNi}_{1-x} \mathrm{Cu}_{x}$ has been extensively studied. ${ }^{6,7} \mathrm{CeCu}$ is antiferromagnetic below $3.5 \mathrm{~K}$ and $\mathrm{CeNi}$ is a nonmagnetic compound with an intermediate valence. The low temperature antiferromagnetic phase changes, around $x=0.8$, to a ferromagnetic one which finally disappears around $x=0.2$. At higher temperatures, a spin-glass state is deduced from all measured bulk properties, such as, for example, the ac susceptibility; for example, for $x=0.6$, the SG state exists between $2 \mathrm{~K}$ and the Curie temperature $T_{c}=1.1 \mathrm{~K}$. At $x=0.2$, there exists below $6 \mathrm{~K}$ only a SG state which transforms to the intermediate valence $\mathrm{CeNi}$, after passing probably through a Kondo lattice system. Thus, in the $\mathrm{CeNi}_{1-x} \mathrm{Cu}_{x}$ system, there appears a SG phase at finite temperatures above the ferromagnetic order and finally a SGKondo transition occurs for small $x$ values.

Another disordered cerium system, namely, $\mathrm{CeCoGe}_{3-x} \mathrm{Si}_{x}$ alloys, has been also studied for different $x$ values, by different experimental techniques including muon spin relaxation. ${ }^{8,9}$ The compound $\mathrm{CeCoGe}_{3}$ is antiferromagnetic below $21 \mathrm{~K}$ and $\mathrm{CeCoSi}_{3}$ is an intermediate valence compound; the QCP of these alloys lies around $x=1.5$. The muon spin relaxation experiments in the quantum critical region ( $x=1.1$ to 1.5$)$ show that a fraction of Ce ions experience random $\mathrm{f}-\mathrm{f}$ indirect exchange interactions, which causes frustration of some Ce spins in the system. So, for $x$ $=1.1$ and $x=1.2$, frustrated moments of cerium freeze like in a spin-glass while the other $\mathrm{Ce}$ moments form a disordered antiferromagnetic state. Thus, near the QCP, a spinglass state can exist in these alloys, in addition to the observed NFL behavior. So, a striking novel behavior, i.e., the appearance of a spin-glass state at finite temperatures in some disordered cerium alloys, has been observed and a SGKondo transition is developing with increasing $x$ around the QCP.

The aim of our paper is to present a theoretical model that describes the spin-glass-Kondo phase transitions, and that we do by studying a system Hamiltonian that couples the localized spins of a Kondo lattice with an additional long range random interaction, like in the Sherrington-Kirkpatrick spinglass model. ${ }^{10} \mathrm{~A}$ similar Hamiltonian has been considered in Ref. 11 to describe NFL behavior and a QCP in some heavy 
fermions compounds, although the relevant approximations differ in this work and ours. The authors in Ref. 11 are primarily interested in the description of the QCP at $T=0$, then they solve first for the Kondo effect by decoupling the conduction electrons bath into independent conduction electron "reservoirs," with no communication between the reservoirs at different sites.

During the course of our work, another paper using the same Hamiltonian has been proposed. ${ }^{12}$ The representation of Popov and Fedotov eliminates the unwanted spin states but the approximations involved are in fact different from those used in our work. They study essentially the spin-glass state and they finally obtain a second order SG transition with a transition temperature depressed by the Kondo effect, in second order perturbation theory.

In the present paper we take a different approach: the localized spins of the Kondo lattice will be effectively immersed in a common bath of conduction electrons and the Kondo effect will be studied in a quantum static approximation that is basically equivalent to the mean field decoupling scheme. ${ }^{3,13}$ The spin operators are represented by bilinear combinations of fermionic creation and destruction operators, for the localized $\mathrm{f}$ electrons, and the spin-glass transition will be studied within the static approximation. This deserves some special discussion. In the Ising quantum spinglass (QSG) model, ${ }^{14}$ the spin operator $S_{i}^{z}$ commutes with the particle number operator $n_{i s}$ and the static ansatz gives the exact answer, as the problem is essentially classic. When we add to the fermionic Ising QSG a s-d exchange coupling of the localized spins to the conduction band electrons the problem ceases to be exactly soluble and the static ansatz is just an approximation that we consider justified to describe a transition at finite temperature. It has been shown in Ref. 15 that the exact numerical solution of Bray and Moore's equations ${ }^{16}$ gives for the spin-spin correlation function $Q(\tau)$ roughly its constant classical value at finite temperature, which justifies the use of the static ansatz of Ref. 16 at not very low temperatures in the Heisenberg spin-glass.

We consider then the static ansatz which corresponds to an approximation similar to mean field theory, whereby neglecting time fluctuations we can provide a description of the phase transitions occurring at finite temperature.

We use functional integral techniques where the spin operators are represented by bilinear combinations of fermionic (anticommuting) Grassmann fields. As we show in the next section, this method is ideally suited to describe a Kondo lattice transition, and it has been recently applied by two of us to the study of fermionic Ising spin-glasses with local BCS pairing. ${ }^{17}$ Recent work ${ }^{18}$ also showed the existence of several characteristic temperatures in the Ising fermionic model, with the de Almeida-Thouless instability ${ }^{19}$ occurring at a temperature lower than the spin-glass transition temperature.

This paper is organized as follows: in Sec. 2 we describe the model and relevant results, we reserve Sec. 3 for discussions and conclusion, while the detailed mathematical calculations are left for the Appendix.

\section{THE MODEL AND RESULTS}

We consider a Kondo lattice system with localized spins $\vec{S}_{i}$ at sites $i=1 \ldots N$, coupled to the electrons of the conduction band via a $\mathrm{s}-\mathrm{d}$ exchange interaction. It is necessary to introduce explicitly the resultant RKKY interaction by means of a random, long range coupling among localized spins like in the Sherrington-Kirkpatrick (SK) model for a spin-glass. To describe the Kondo effect in a mean-field-like theory it is sufficient to keep only the spin-flip terms ${ }^{3}$ in the exchange Hamiltonian, while the spin-glass interaction is represented by the quantum Ising Hamiltonian where only the $z$ components of the localized spins interact. ${ }^{11,14,17}$

The Hamiltonian of the model is

$$
\begin{array}{r}
\mathcal{H}-\mu_{c} N_{c}-\mu_{f} N_{f}=\mathcal{H}_{k}-\mu_{c} N_{c}-\mu_{f} N_{f}+\mathcal{H}_{\mathrm{SG}}, \\
\mathcal{H}_{k}-\mu_{c} N_{c}-\mu_{f} N_{f}=\sum_{k, \sigma} \epsilon_{k} n_{k \sigma}+\epsilon_{0} \sum_{i, \sigma} n_{i \sigma}^{f} \\
+J_{k} \sum_{i}\left[S_{f i}^{+} s_{c i}^{-}+S_{f i}^{-} s_{c i}^{+}\right], \\
\mathcal{H}_{\mathrm{SG}}=-\sum_{i, j} J_{i j} S_{f i}^{z} S_{f j}^{z}-2 h_{0} \sum_{i} S_{f i}^{z},
\end{array}
$$

where $J_{k}>0$,

$$
\begin{gathered}
S_{f i}^{+}=f_{i \uparrow}^{\dagger} f_{i \downarrow} ; \quad s_{c i}^{+}=d_{i \uparrow}^{\dagger} d_{i \downarrow}, \\
S_{f i}^{-}=f_{i \downarrow}^{\dagger} f_{i \uparrow} ; \quad s_{c i}^{-}=d_{i \downarrow}^{\dagger} d_{i \uparrow}, \\
S_{f i}^{z}=\frac{1}{2}\left[f_{i \uparrow}^{\dagger} f_{i \uparrow}-f_{i \downarrow}^{\dagger} f_{i \downarrow}\right],
\end{gathered}
$$

and $f_{i \sigma}^{+}, f_{i \sigma}\left(d_{i \sigma}^{+}, d_{i \sigma}\right)$ are creation and destruction operators for electrons with spin projection $\sigma=\uparrow$ or $\downarrow$ in the localized (conduction) band, that satisfy the standard fermion anticommutation rules. We also have $n_{k \sigma}=d_{k \sigma}^{+} d_{k \sigma}$ where

$$
\begin{aligned}
& d_{k \sigma}=\frac{1}{\sqrt{N}} \sum_{i} e^{i \vec{k} \cdot \vec{R}_{i}} d_{i \sigma}, \\
& d_{k \sigma}^{+}=\frac{1}{\sqrt{N}} \sum_{i} e^{-i \vec{k} \cdot \vec{R}_{i}} d_{i \sigma}^{+} .
\end{aligned}
$$

The energies $\epsilon_{0}\left(\epsilon_{k}\right)$ are referred to the chemical potentials $\mu_{f}\left(\mu_{c}\right)$, respectively.

The coupling $J_{i j}$ in Eq. (3) is an independent random variable with the gaussian distribution

$$
P\left(J_{i j}\right)=e^{-J_{i j}^{2}\left(N / 32 J^{2}\right)} \sqrt{\frac{N}{32 \pi J^{2}}} .
$$

Functional integration techniques have proved to be a suitable approach to describe phase transitions in disordered quantum mechanical many-particle systems. ${ }^{20}$ The static approximation within this formulation consists in neglecting time fluctuations of the order parameter, and when it is combined with the neglect of space fluctuations it leads to the usual Hartree-Fock, mean field like approximation. When dealing with the Hamiltonian in Eqs. (1)-(3), we notice that in the limiting case $J_{k}=0$ we obtain a pure quantum Ising 
spin-glass where the static approximation gives the exact result, ${ }^{14,18}$ while for $J=0$ we recover the mean field approximation that has been used successfully to describe the Kondo lattice. ${ }^{3,13}$ Then we consider that the use of the static approximation has an interpolation character and will provide reliable results to describe critical behavior at finite temperature $^{15}$ in systems that do not present a quantum critical point.

In the Lagrangian formulation ${ }^{17,20}$ the partition function is expressed as

$$
Z=\int D\left(\varphi^{\dagger} \varphi\right) D\left(\psi^{\dagger} \psi\right) e^{A}
$$

where the action $A$ is given by

$$
A=\sum_{i, \sigma} \int_{0}^{\beta} d \tau\left\{\left(\frac{\partial}{\partial \tau} \varphi_{i \sigma}^{\dagger}\right) \varphi_{i \sigma}+\left(\frac{\partial}{\partial \tau} \psi_{i \sigma}^{\dagger}\right) \psi_{i \sigma}\right\}-\int_{0}^{\beta} \mathcal{H}(\tau) d \tau
$$

In both expressions $\varphi_{i \sigma}(\tau)$ and $\psi_{i \sigma}(\tau)$ are anticommuting, complex Grassmann variables associated to the conduction and localized electrons fields, respectively, while $\tau$ is an imaginary time and $\beta$ the inverse absolute temperature.

We show in the Appendix that in the static, mean field like approximation the action A may be written

$$
A=A_{0}+A_{K}+A_{\mathrm{SG}},
$$

with

$$
\begin{aligned}
A_{0}= & \sum_{\omega \sigma} \sum_{i, j}\left[\left(i \omega-\beta \epsilon_{0}\right) \delta_{i j} \psi_{i \sigma}^{\dagger}(\omega) \psi_{i \sigma}(\omega)\right. \\
& \left.+\left(i \omega \delta_{i j}-\beta t_{i j}\right) \varphi_{i \sigma}^{\dagger}(\omega) \varphi_{j \sigma}(\omega)\right],
\end{aligned}
$$

where from Eq. (A2)

$$
\begin{gathered}
A_{K}=\frac{\beta J_{k}}{N} \sum_{\sigma}\left[\sum_{i \omega} \psi_{i, \sigma}^{\dagger}(\omega) \varphi_{i \sigma}(\omega)\right]\left[\sum_{i, \omega} \varphi_{i-\sigma}^{\dagger} \psi_{i-\sigma}(\omega)\right] \\
A_{\mathrm{SG}}=\sum_{i, j} J_{i j} S_{i}^{z} S_{j}^{z}-2 h_{0} \sum_{i} S_{f i}^{z}
\end{gathered}
$$

and in the static approximation ${ }^{14,17,18}$

$$
S_{i}^{z}=\frac{1}{2} \sum_{\sigma} \sum_{\omega} \psi_{i \sigma}^{\dagger}(\omega) \psi_{i \sigma}(\omega)
$$

The sums are over fermion Matsubara frequencies $\omega=(2 n$ $+1) \pi$.

The Kondo order is described by the complex order parameter

$$
\begin{aligned}
& \lambda_{\sigma}^{\dagger}=\frac{1}{N} \sum_{i, \omega}\left\langle\psi_{i \sigma}^{\dagger}(\omega) \varphi_{i \sigma}(\omega)\right\rangle, \\
& \lambda_{\sigma}=\frac{1}{N} \sum_{i, \omega}\left\langle\varphi_{i \sigma}^{\dagger}(\omega) \psi_{i \sigma}(\omega)\right\rangle,
\end{aligned}
$$

that in a mean field theory ${ }^{3,13}$ describes the correlations $\lambda_{\sigma}^{\dagger}$ $=\left\langle f_{i \sigma}^{\dagger} d_{i \sigma}\right\rangle$ and $\lambda_{\sigma}=\left\langle d_{i \sigma}^{\dagger} f_{i \sigma}\right\rangle$. Complex conjugation of Grassmann variables is defined through the transposition $\operatorname{rule}^{20}\left(\psi^{\dagger} \varphi\right)^{\dagger}=\varphi^{\dagger} \psi$.

We show in the Appendix that standard manipulations give for the averaged free energy within a replica symmetric theory:

$$
\beta F=2 \beta J_{k} \lambda^{2}+\frac{1}{2} \beta^{2} J^{2}\left(\bar{\chi}^{2}+2 q \bar{\chi}\right)-\beta \Omega,
$$

where

$$
\begin{aligned}
\beta \Omega= & \lim _{n \rightarrow 0} \frac{1}{N n}\left\{\int_{-\infty}^{+\infty} \prod_{j}^{N} D z_{j} \prod_{\alpha}^{n} \int_{-\infty}^{+\infty} \prod_{j} D \varepsilon_{\alpha j}\right. \\
& \left.\times \exp \left(\sum_{\omega, \sigma} \ln \left|G_{i j \sigma}^{-1}(\omega)\right|\right)-1\right\},
\end{aligned}
$$

and the order parameters $q, \bar{\chi}$, and $\lambda$ must be taken at their saddle point value. Here $q$ is the SG order parameter ${ }^{14,17,18}$ and $\chi=\beta \bar{\chi}$ is the static uniform spin susceptibility of the localized $\mathrm{f}$ electrons. We use the notation $D x$ $=(d x / \sqrt{2 \pi}) e^{-(1 / 2) x^{2}}$.

The function $G_{i j \sigma \alpha}(\omega)$ in Eq. (16) is the time Fourier transform of the Green's function $G_{i j \sigma}(\tau)$ $=i\left\langle T f_{i \sigma}(\tau) f_{j \sigma}^{\dagger}(0)\right\rangle$ for the localized electrons in the presence of random fields $z_{j}$ and $\varepsilon_{\alpha j}$ at every site, and from Eq. (A23) satisfies the equation

$$
G_{i j \sigma \alpha}^{-1}(\omega)=\left[i \omega-\beta \epsilon_{0}-\sigma h_{j \alpha}\right] \delta_{i j}-\beta^{2} J_{k}^{2} \lambda^{2} \gamma_{i j}(\omega),
$$

where

$$
h_{j \alpha}=\sqrt{2 q} \beta J z_{j}+\sqrt{2 \bar{\chi}} \beta J \varepsilon_{\alpha j}+h_{0},
$$

while $\gamma_{i j}(\omega)$ is the time Fourier transform of the conduction electron Green's function $\gamma_{i j}(\tau)=i\left\langle T d_{i \sigma}(\tau) d_{i \sigma}^{\dagger}(0)\right\rangle$ and is given by

$$
\gamma_{i j}^{-1}=\left[i \omega-\beta \mu_{c}\right] \delta_{i j}-\beta t_{i j}
$$

We obtained in Eq. (17) the Green's function for the $\mathrm{f}$ electrons in a Kondo lattice, ${ }^{13}$ but now in the presence of a random field $h_{j}$ at every site that prevents us from proceeding with the calculation. In the pure $\mathrm{SG}$ limit $J_{k}=0$ the Green's function in Eq. (17) is local and the integrals in Eq. (16) reduce to a one site problem, while in the Kondo limit $J=0$ the random fields vanish and the integrals separate in reciprocal space. We adopt here a decoupling approximation that is reminiscent of the model with independent "reservoirs" of electrons considered in Ref. 11. We replace the Green's function $G_{i j \sigma \alpha}\left(\omega,\left\{h_{1 \alpha} \ldots h_{j \alpha} \ldots h_{N \alpha}\right\}\right)$ by the Green's functions $\Gamma_{\mu \nu \sigma}\left(\omega, h_{j \alpha}\right), j=1 \ldots N$, of $N$ independent Kondo lattices, each one with a "uniform" field $h_{j \alpha}$ at every site $\mu, \nu$, by means of the approximation 


$$
\ln \left|G_{i j \sigma \alpha}^{-1}\left(\omega,\left\{h_{1} \ldots h_{N}\right\}\right)\right| \approx \frac{1}{N} \sum_{j} \ln \left|\Gamma_{\mu \nu \sigma}^{-1}\left(\omega, h_{j \alpha}\right)\right|,
$$

where $\Gamma_{\mu \nu \sigma}\left(\omega, h_{j \alpha}\right)$ is the $\mathrm{f}$ electron Green's function for a fictitious Kondo lattice that has a uniform field $h_{j \alpha}$ at every site $\mu, \nu$ and satisfies the equation

$$
\Gamma_{\mu \nu \sigma}^{-1}\left(\omega, h_{j}\right)=\left[i \omega-\beta \epsilon_{0}-\sigma h_{j \alpha}\right] \delta_{\mu \nu}-\beta^{2} J_{k}^{2} \lambda^{2} \gamma_{\mu \nu},
$$

where, from Eq. (19),

$$
\gamma_{\mu \nu}(\omega)=\frac{1}{N} \sum_{k} \frac{1}{i \omega-\beta \epsilon_{k}} e^{i \vec{k} \cdot \vec{R}_{\mu \nu}}
$$

Now Eq. (21) may be easily solved by a Fourier transformation with the result

$$
\ln \left|\Gamma_{\mu \nu \sigma}^{-1}\left(\omega, h_{j \alpha}\right)\right|=\sum_{\vec{k}} \ln \left[\Gamma_{\vec{k} \sigma}^{-1}\left(\omega, h_{j \alpha}\right)\right]
$$

where

$$
\Gamma_{\vec{k} \sigma}^{-1}\left(\omega, h_{j \alpha}\right)=\left[i \omega-\beta \epsilon_{0}-\sigma h_{j \alpha}\right]-\beta^{2} J_{k}^{2} \lambda^{2} \frac{1}{i \omega-\beta \epsilon_{k}} .
$$

We may now introduce Eqs. (20) and (23) in Eq. (16), the integrals over the fields separate and we obtain

$$
\beta \Omega=\int_{-\infty}^{+\infty} D z \ln \left\{\int_{-\infty}^{+\infty} D \varepsilon \exp \left(\sum_{\sigma} \frac{1}{N} \sum_{\vec{k}} S_{\sigma}(\vec{k}, h)\right)\right\},
$$

with

$$
S_{\sigma}(\vec{k}, h)=\sum_{\omega} \ln \left[\Gamma_{\vec{k}, \sigma}^{-1}(\omega, h)\right]
$$

and $h$ is given in Eq. (18), with $z$ and $\varepsilon$ in place of $z_{j}$ and $\varepsilon_{j \alpha}$.

The sum over the fermion frequencies is performed in the standard way by integrating in the complex plane, ${ }^{20}$ with the result

$$
S_{\sigma}(\vec{k}, h)=\ln \left[\left(1+e^{-\omega_{\sigma^{+}}}\right)\left(1+e^{-\omega_{\sigma^{-}}}\right)\right]
$$

where

$$
\omega_{\sigma^{ \pm}}=\frac{1}{2}\left[\beta \epsilon_{k}+\sigma h\right] \pm\left\{\frac{1}{4}\left(\beta \epsilon_{k}-\sigma h\right)^{2}+\left(\beta J_{k} \lambda\right)^{2}\right\}^{1 / 2} .
$$

We consider $\epsilon_{0}=0$ that corresponds to an average occupation $\left\langle n_{f}\right\rangle=1$, per site.

Replacing sums by integrals, in the approximation of a constant density of states for the conduction band electrons, $\rho(\epsilon)=\rho=1 /(2 D)$ for $-D<\epsilon<D$, we obtain from Eqs. (25) to (27) the final expression for the free energy in Eq. (15)

$$
\begin{aligned}
\beta F= & 2 \beta J_{k} \lambda^{2}+\frac{1}{2} \beta^{2} J^{2}\left(\bar{\chi}^{2}+2 q \bar{\chi}\right) \\
& -\int_{-\infty}^{+\infty} D z \ln \left\{\int_{-\infty}^{+\infty} D \varepsilon e^{E(h)}\right\},
\end{aligned}
$$

with

$$
E(h)=\frac{1}{\beta D} \int_{-\beta D}^{+\beta D} d x \ln \left\{\cosh \frac{(x+h)}{2}+\cosh (\sqrt{\Delta})\right\},
$$

$$
\Delta=\frac{1}{4}(x-h)^{2}+\left(\beta J_{k} \lambda\right)^{2},
$$

and from Eq. (18) we have $h=\beta J[\sqrt{2 q} z+\sqrt{2 \bar{\chi}} \varepsilon]$. The saddle point equations for the SG order parameters are

$$
q=\int_{-\infty}^{+\infty} D z\left\{\frac{\int D \varepsilon e^{E}(\partial E / \partial h)}{\int D \varepsilon e^{E}}\right\}^{2},
$$

$$
\bar{\chi}=\int_{-\infty}^{+\infty} D z \frac{1}{\int D \varepsilon e^{E}}\left\{\int D \varepsilon \frac{\partial}{\partial h}\left[e^{E} \frac{\partial E}{\partial h}\right]\right\},
$$

while we obtain for the Kondo order parameter $\lambda$

$$
\begin{aligned}
4 \beta J_{k} \lambda & \left\{1-\frac{\beta J_{k}}{4} \int_{-\infty}^{+\infty} D z \frac{1}{\int D \varepsilon e^{E}} \int D \varepsilon e^{E} \frac{1}{\beta D}\right. \\
& \left.\times \int_{-\beta D}^{+\beta D} d x \frac{1}{\cosh \left(\frac{x+h}{2}\right)+\cosh (\sqrt{\Delta})}\left(\frac{\sinh (\sqrt{\Delta})}{\sqrt{\Delta}}\right)\right\} \\
= & 0 .
\end{aligned}
$$

The numerical solution of the saddle point equations when $h_{0}=0$ as a function of $T / J$ and $J_{k} / J$ provides us with the phase diagram in Fig. 1, that we discuss in the next section.

\section{CONCLUSIONS}

We study in this paper the phase transitions in a system represented by a Hamiltonian that couples the localized spins of a Kondo lattice ${ }^{3,13}$ with random, long range interactions, like in the SK model for a spin-glass. ${ }^{10}$

Using functional integrals techniques and a static, replica symmetric ansatz for the Kondo and spin-glass order parameters, we derive a mean field expression for the free energy and the saddle point equations for the order parameters. The Kondo and spin-glass transitions are both described with the mean field like static ansatz that reproduces good results in two well-known limits: when $J_{k}=0$ we recover the exact solution for the quantum Ising spin-glass, ${ }^{14,18}$ while for $J$ $=0$ we recover the mean field results for the Kondo 


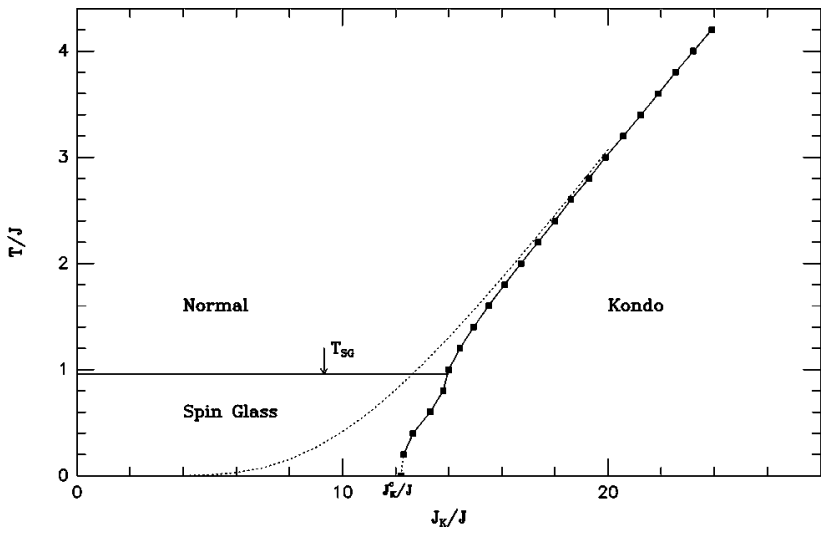

FIG. 1. Phase diagram in the $T-J_{k}$ plane as a function of $T / J$ and $J_{k} / J$ for fixed $J=0.05 D$, where $D$ is the conduction bandwidth. The dotted line represents the "pure" Kondo temperature $T_{k}$.

lattice. ${ }^{3,13}$ The use of the static ansatz is justified at finite temperatures. ${ }^{15}$ Numerical solution of the saddle-point equations allow us to draw the magnetic phase diagram in the $J_{k}$ vs $T$ plane, for fixed value of $J$, presented in Fig. 1 .

Figure 1 shows three different phases. At high temperatures, the "normal" phase is paramagnetic with vanishing Kondo and spin-glass order parameters, i.e., $\lambda=q=0$. When temperature is lowered, for not too large values of the ratio $J_{K} / J$, a second-order transition line is found at $T=T_{\mathrm{SG}}$ to a spin-glass phase with $q>0$ and $\lambda=0$. Finally, for large values of the ratio $J_{K} / J$, we recover the "Kondo" phase with a nonzero $\lambda$ value and $q=0$ : the transition line from the paramagnetic phase to the Kondo phase for temperatures larger than $T_{\mathrm{SG}}$ is a second-order one and occurs at a temperature very close to the one-impurity Kondo temperature $T_{K}$. On the other hand, the transition line from the spin-glass phase to the Kondo phase, for temperatures smaller than $T_{\mathrm{SG}}$, is a first-order one and it ends at $J_{K}^{c}$ at $T=0$. When the temperature is lowered, the transition temperature does not vary very much with the value of $J_{K} / J$; the separation between the spin-glass and the Kondo phases departs completely from the behavior of $T_{K}$ and looks like the separation between the magnetic and Kondo phases when these two phases are considered. $^{21}$ We can also remark that we get here only "pure" Kondo or SG phases and never a mixed SG-Kondo phase with the two order parameters different from zero; this result is probably connected to the approximations used here to treat the starting Hamiltonian.

The diagram shown in Fig. 1 can explain the magnetic phase diagram observed above the Curie temperature for the $\mathrm{CeNi}_{1-x} \mathrm{Cu}_{x}$ (Ref. 6) for small $x$ values when there is a transition from a spin-glass state to a Kondo state and then to the intermediate valence compound $\mathrm{CeNi}$; however, there is no experimental information on the precise nature of the SGKondo transition and our model cannot be checked from that point of view. There is also probably a SG-Kondo transition in the $\mathrm{CeCoGe}_{3-x} \mathrm{Si}_{x}$ alloys, but there the experimental situation is even more complicated than in the preceding case. An unsolved basic question concerns also the existence or not of a "mixed" SG-Kondo phase in cerium disordered alloys and this problem is worthy of being studied experi- mentally in more detail. Thus, further experimental work is necessary, but our model yields a new striking point in the behavior of heavy fermion disordered alloys in the vicinity of the quantum critical point.

\section{ACKNOWLEDGMENTS}

We acknowledge enlightening discussions with J. C. Gómez Sal. This work was partially supported by Conselho Nacional de Pesquisa e Desenvolvimento (CNPQ) and by Financiadora de Estudos e Projetos (FINEP).

\section{APPENDIX}

We present here a detailed derivation of the main equations of the paper. By introducing Eqs. (1)-(3) in Eq. (8) we obtain for the $\mathrm{s}-\mathrm{d}$ exchange part of the action:

$$
\begin{aligned}
A_{K}= & -\beta J_{k} \sum_{i} \sum_{\omega, \omega^{\prime}, \Omega} \sum_{\sigma} \psi_{i \sigma}^{\dagger}(\omega) \psi_{i-\sigma}\left(\omega^{\prime}+\Omega\right) \\
& \times \varphi_{i-\sigma}^{\dagger}\left(\omega^{\prime}\right) \varphi_{i \sigma}(\omega-\Omega),
\end{aligned}
$$

where $\omega=(2 n+1) \pi$ and $\Omega=2 n \pi$. In the mean field spirit we want to introduce the spatially uniform and static Kondo order parameter in Eq. (14), then we take $\Omega=0$, reorder the operators and separate the sites in Eq. (A1) with the introduction of an $N^{-1}$ factor, which gives

$A_{K} \approx+\frac{\beta J_{k}}{N} \sum_{\sigma} \sum_{i, \omega} \psi_{i \sigma}^{\dagger}(\omega) \varphi_{i \sigma}(\omega) \sum_{j, \omega^{\prime}} \varphi_{j-\sigma}^{\dagger}\left(\omega^{\prime}\right) \psi_{j-\sigma}\left(\omega^{\prime}\right)$,

that is Eq. (11).

We find it convenient to introduce the Kondo order parameters in Eq. (14) by means of the identity

$$
\begin{aligned}
e^{A_{k}=} & \int_{-\infty}^{\infty} \Pi_{\sigma} d \lambda_{\sigma}^{\dagger} d \lambda_{\sigma} \Pi_{\sigma} \\
& \times \delta\left[\lambda_{\sigma}^{\dagger} N-\sum_{j, \omega} \psi_{j \sigma}^{\dagger}(\omega) \varphi_{j \sigma}(\omega)\right] \\
& \times \delta\left[\lambda_{\sigma} N-\sum_{j, \omega} \varphi_{j \sigma}^{\dagger}(\omega) \psi_{j \sigma}(\omega)\right] \\
& \times e^{\beta J_{k} N\left[\lambda_{\uparrow}^{\dagger} \lambda_{\downarrow}+\lambda_{\downarrow}^{\dagger} \lambda_{\uparrow}\right]},
\end{aligned}
$$

and using the integral representation of the $\delta$ function

$$
\delta\left(x-x_{0}\right)=\frac{1}{2 \pi} \int_{-\infty}^{\infty} d u e^{i u\left(x-x_{0}\right)}
$$

we may write the partition function by combining Eqs. (7) to (13),

$$
\begin{aligned}
Z= & \int_{-\infty}^{\infty} \Pi_{\sigma} d \lambda_{\sigma}^{\dagger} d \lambda_{\sigma} \int_{-\infty}^{\infty} \Pi_{\sigma} \frac{d u_{\sigma} d v_{\sigma}}{(2 \pi)^{2}} \\
& \times e^{N \Sigma_{\sigma}\left\{\beta J_{k} \lambda_{\sigma}^{\dagger} \lambda_{-\sigma}-i\left[u_{\sigma} \lambda_{\sigma}^{\dagger}+v_{\sigma} \lambda_{\sigma}\right]\right\}} Z_{\text {eff }},
\end{aligned}
$$


where

$$
\begin{aligned}
Z_{\mathrm{eff}}= & \int D\left(\psi^{\dagger} \psi\right) \int D\left(\varphi^{\dagger} \varphi\right) e^{A_{0}+A_{\mathrm{SG}}} \\
& \times e^{\Sigma_{\sigma} i\left[v_{\sigma} \Sigma_{j, \omega} \varphi_{j \sigma}^{\dagger}(\omega) \psi_{j \sigma}(\omega)+u_{\sigma} \Sigma_{j, \omega} \psi_{j \sigma}^{\dagger}(\omega) \varphi_{j \sigma}(\omega)\right]} .
\end{aligned}
$$

The saddle point values of $\lambda_{\sigma}, \lambda_{\sigma}^{\dagger}, u_{\sigma}, u_{\sigma}^{\dagger}$ are obtained by extremizing the exponent in Eq. (A5)

$$
\begin{gathered}
\beta J_{k} \lambda_{-\sigma}^{\dagger}=i v_{\sigma}, \\
\beta J_{k} \lambda_{-\sigma}=i u_{\sigma}, \\
\lambda_{\sigma}^{\dagger}=\frac{1}{N} \sum_{j, \omega}\left\langle\psi_{j \sigma}^{\dagger}(\omega) \varphi_{i \sigma}(\omega)\right\rangle, \\
\lambda_{\sigma}=\frac{1}{N} \sum_{j, \omega}\left\langle\varphi_{i \sigma}^{\dagger}(\omega) \psi_{j \sigma}(\omega)\right\rangle,
\end{gathered}
$$

where $\langle\cdots\rangle=\int D\left(\psi^{\dagger} \psi\right) D\left(\varphi^{\dagger} \varphi\right) e^{A_{\text {eff }}(\cdots)}$ from Eq. (A6). Introducing the saddle point values of Eq. (A7) into Eq. (A5), we obtain

$$
Z=e^{-2 N \beta J_{k} \lambda^{\dagger} \lambda} Z_{\text {eff }}
$$

From Eq. (10), $Z_{\text {eff }}$ is now the integral of a quadratic form in the $\varphi^{\dagger}, \varphi$ variables, that can be integrated out to give

$$
Z_{\mathrm{eff}}=Z_{d}^{0} Z_{\mathrm{SG}},
$$

where $Z_{d}^{0}$ is the partition function of the free conducting electrons,

$$
\ln \left(Z_{d}^{0}\right)=2 \sum_{\omega} \log \left|\gamma_{i j}^{-1}(\omega)\right|
$$

and

$$
\gamma_{i j}^{-1}(\omega)=i \omega \delta_{i j}-\beta t_{i j}
$$

is the inverse Green's function for the d electrons. The quantity $Z_{\mathrm{SG}}$ in Eq. (A9) is the partition function for the localized f electrons,

$$
Z_{\mathrm{SG}}=\int D\left(\psi^{\dagger} \psi\right) e^{\Sigma_{\omega \sigma} \Sigma_{i, j} g_{i j}^{-1}(\omega) \psi_{i \sigma}^{\dagger}(\omega) \psi_{j \sigma}(\omega)+A_{\mathrm{SG}}},
$$

where the inverse Green's function for the localized, noninteracting $\mathrm{f}$ electrons is now modified by the Kondo interaction,

$$
g_{i j}^{-1}(\omega)=\left(i \omega-\beta \varepsilon_{0}\right) \delta i j-\beta^{2} J_{k}^{2} \lambda^{\dagger} \lambda \gamma_{i j}(\omega)
$$

and $A_{\mathrm{SG}}$ is given in Eq. (12).

The interesting part of the free energy is given by

$$
\beta F=-\frac{1}{N}\left\langle\left\langle\ln \left(\frac{Z}{Z_{d}^{0}}\right)\right\rangle\right\rangle
$$

where the double bracket indicates a configurational average over the random variables $J_{i j}$, with the distribution probability in Eq. (16). Using the replica method we obtain from Eqs. (A8) and (A9)

$$
\beta F=2 \beta J_{k} \lambda^{\dagger} \lambda-\lim _{n \rightarrow 0} \frac{1}{N n}\left[Z_{n}(\mathrm{SG})-1\right],
$$

where $\alpha=1 \ldots n$ is the replica index and

$$
\begin{aligned}
Z_{n}(\mathrm{SG})= & \left\langle\left\langle Z_{\mathrm{SG}}^{n}\right\rangle\right\rangle=\int \prod_{\alpha}^{n} D\left(\psi_{\alpha}^{\dagger} \psi_{\alpha}\right) \\
& \times \exp \left\{\sum_{\omega \sigma} \sum_{i, j} g_{i j}^{-1}(\omega) \sum_{\alpha} \psi_{i \sigma \alpha}^{\dagger}(\omega) \psi_{j \sigma \alpha}(\omega)\right\} \\
& \times \prod_{i, j}\left\langle\left\langle e^{\left.\beta J_{i j} \Sigma_{\alpha} S_{i \alpha}^{z} S_{j \alpha}^{z}\right\rangle}\right\rangle\right.
\end{aligned}
$$

The operators $S_{i \alpha}^{z}$ are bilinear combinations of $\psi_{i \sigma \alpha}^{\dagger}(\omega)$, $\psi_{i \sigma \alpha}(\omega)$ from Eq. (13), then after performing the average in Eq. (A16) we must use standard manipulations with gaussian identities ${ }^{16,17}$ to linearize the exponent in Eq. (A16). We obtain

$$
Z_{n}(\mathrm{SG})=\int \prod_{\alpha, \beta} d q_{\alpha \beta} e^{-(1 / 2)(\beta J)^{2} N \Sigma_{\alpha, \beta} q_{\alpha \beta}^{2} \Lambda\left(\left\{q_{\alpha \beta}\right\}\right),}
$$

where

$$
\begin{aligned}
\Lambda\left(\left\{q_{\alpha \beta}\right\}\right)= & \int D\left(\psi_{\alpha}^{\dagger} \psi_{\alpha}\right) \\
& \times \exp \left\{\sum_{i, j} \sum_{\omega, \sigma, \alpha} g_{i j}^{-1}(\omega) \psi_{i \sigma \alpha}^{\dagger}(\omega) \psi_{j \sigma \alpha}(\omega)\right. \\
& \left.+\beta^{2} J^{2} \sum_{\alpha, \beta} q_{\alpha \beta} \sum_{i} S_{i \alpha}^{z} S_{i \beta}^{z}\right\}
\end{aligned}
$$

We obtain for $Z_{n}(\mathrm{SG})$ at the replica symmetric saddle point

$$
\begin{gathered}
q_{\alpha \neq \beta}=q=\lim _{n \rightarrow 0} \frac{1}{n(n-1)} \sum_{\alpha \neq \beta}\left\langle S_{\alpha}^{z} S_{\beta}^{z}\right\rangle, \\
q_{\alpha \alpha}=q+\bar{\chi}=\lim _{n \rightarrow 0} \frac{1}{n} \sum_{\alpha}\left\langle S_{\alpha}^{z} S_{\alpha}^{z}\right\rangle, \\
Z_{n}(\mathrm{SG}) \approx e^{-(1 / 2)(\beta J)^{2} N\left(\bar{\chi}^{2}+2 q \bar{\chi}\right)} \Lambda(q, \bar{\chi}),
\end{gathered}
$$

where

$$
\Lambda(q, \bar{\chi})=\int_{-\infty}^{\infty} \prod_{j} D z_{j} \prod_{\alpha} \int_{-\infty}^{\infty} \prod_{j} D \xi_{\alpha j} I_{\alpha}\left(q, \bar{\chi},\left\{z_{j}\right\},\left\{\xi_{\alpha j}\right\}\right)
$$

and 


$$
\begin{aligned}
& I_{\alpha}\left(q, \bar{\chi},\left\{z_{j}\right\},\left\{\xi_{\alpha j}\right\}\right) \\
& \quad=\int D\left(\psi_{\alpha}^{\dagger} \psi_{\alpha}\right) \exp \left\{\sum_{\omega, \sigma} \sum_{i, j} G_{i j \sigma \alpha}^{-1}(\omega) \psi_{i \sigma \alpha}^{\dagger}(\omega) \psi_{j \sigma \alpha}(\omega)\right\} \\
& \quad=e^{\Sigma_{\omega, \sigma} \ln \left|G_{i j \sigma \alpha}^{-1}(\omega)\right|},
\end{aligned}
$$

where, making $h_{0}=0$

$$
G_{i j \sigma \alpha}^{-1}(\omega)=g_{i j}^{-1}(\omega)-\delta_{i j} \sigma\left[\sqrt{2 q} \beta J z_{j}+\sqrt{2 \bar{\chi}} \beta J \xi_{\alpha j}\right] .
$$

Introducing Eqs. (A23) and (A22) into Eq. (A21), we obtain from Eq. (A15) the expression for the free energy in Eq. (15) of the main text.
The order parameter $\bar{\chi}$ in Eq. (A20) is related to the uniform static susceptibility $\chi$ of the localized f electrons

$$
\chi=\left.\frac{1}{N^{2} \beta} \frac{\partial^{2}\langle\ln Z\rangle}{\partial h_{o}^{2}}\right|_{h_{o}=0}=\beta \bar{\chi},
$$

as it was discussed in our previous publications. ${ }^{14,18}$ In the Sherrington-Kirkpatrick model ${ }^{10}$ we have the identity $q_{\alpha \alpha}^{\mathrm{SK}}$ $=1$ and Eq. (A20) reduces to the known expression $\chi_{\mathrm{SK}}$ $=\beta(1-q)$, while in the present model the occupation number fluctuates and $\bar{\chi}$ must be determined self-consistently.
${ }^{1}$ S. Doniach, Physica B 91, 231 (1977).

${ }^{2}$ T. Graf, J.D. Thompson, M.F. Hundley, R. Movshovich, Z. Fisk, D. Mandrus, R.A. Fischer, and N.E. Phillips, Phys. Rev. Lett. 78, 3769 (1997).

${ }^{3}$ J.R. Iglesias, C. Lacroix, and B. Coqblin, Phys. Rev. B 56, 11820 (1997).

${ }^{4}$ Proceedings of the Conference of NFL Behavior in Metals, Santa Barbara, 1996, edited by P. Coleman, M.B. Maple, and A.J. Millis [J. Phys.: Condens. Matter 8, 9675 (1996)].

${ }^{5}$ B. Coqblin, B.H. Bernard, J.R. Iglesias, C. Lacroix, and K. Le Hur, in Electron Correlations and Materials Properties, edited by Gonis et al. (Kluwer Academic/Plenum, New York, 1999), pp. 225-250.

${ }^{6}$ J. Garcia Soldevilla, J.C. Gomez Sal, J.A. Blanco, J.I. Espeso, and J. Rodriguez Fernandez, Phys. Rev. B 61, 6821 (2000).

${ }^{7}$ J. C. Gómez Sal, J. Garcia Soldevilla, J.A. Blanco, J.I. Espeso, J. Rodriguez Fernandez, F. Luis, F. Bartolomé, and J. Bartolomé, Phys. Rev. B 56, 11741 (1997).

${ }^{8}$ D. Eom, M. Ishikawa, J. Kitagawa, and N. Takeda, J. Phys. Soc. Jpn. 67, 2495 (1998).

${ }^{9}$ V.V. Krishnamurthy, K. Nagamine, I. Watanabe, K. Nishiyama, S. Ohira, M. Ishikawa, D. Eom, and T. Ishikawa (unpublished).
${ }^{10}$ D. Sherrington and S. Kirkpatrick, Phys. Rev. Lett. 35, 1792 (1975).

${ }^{11}$ Anirvan M. Sengupta and Antoine Georges, Phys. Rev. B 52, 10295 (1995).

${ }^{12}$ M.N. Kiselev and R. Oppermann, Pis'ma Zh. Eksp. Teor. Fiz. 71, 359 (2000).

${ }^{13}$ C. Lacroix and M. Cyrot, Phys. Rev. B 20, 1969 (1979).

${ }^{14}$ (a) Alba Theumann and M. Vieira Gusmão, Phys. Lett. 105A, 311 (1984); (b) Alba Theumann, Phys. Rev. B 33, 559 (1986).

${ }^{15}$ D.R. Grempel and M.J. Rozenberg, Phys. Rev. Lett. 80, 389 (1998).

${ }^{16}$ A.J. Bray and M.A. Moore, J. Phys. C 13, 419 (1980).

${ }^{17}$ S.G. Magalhães and Alba Theumann, Eur. Phys. J. B 9, 5 (1999).

${ }^{18}$ Alba Theumann, Phys. Rev. B 56, 5500 (1997).

${ }^{19}$ J.R.L. Almeida and D.J. Thouless, J. Phys. A 11, 983 (1978).

${ }^{20}$ (a) John W. Negele and Henry Orland, Quantum Many Particles (Addison-Wesley, Reading, MA, 1987); (b) V.N. Popov, Functional Integrals and Collective Excitations (Cambridge University Press, Cambridge, 1987).

${ }^{21}$ F. Duhem and C. Lacroix (unpublished); A. Ruppenthal, Ph.D. thesis, Universidade Federal do Rio Grande do Sul, Brazil, 1999. 